http://jmscr.igmpublication.org/home/ ISSN (e)-2347-176x ISSN (p) 2455-0450

crossref DOI: https://dx.doi.org/10.18535/jmscr/v8i9.14

Journal Of Medical Science And Clinical Research

IGM Publication

An official Publication of IGM Publication

\title{
External Quality Assessment of Haemoglobin Measurement: A Comparison of Different Analysers
}

\author{
Authors \\ Esther Wangui Mandania, MMLS ${ }^{1 *}$, Juliette R. Ongus, Ph.D. ${ }^{2}$, \\ Sarah Njoki Kaggia, MMed ${ }^{3}$, Fatmah Abdallah, MMed ${ }^{4}$
}

${ }^{1}$ Department of Medical Laboratory Sciences, Medical School, College of Health Sciences, Mount Kenya University

${ }^{2}$ Department of Medical Laboratory Sciences, Jomo Kenyatta University of Agriculture and Technology

${ }^{3}$ Department of Human Pathology, Jomo Kenyatta University of Agriculture and Technology

${ }^{4}$ Department of human pathology, Haematology and Blood Transfusion Unit, College of Health Sciences, University Of Nairobi

Department the work should be attributed to: Department of Medical Laboratory Sciences, Medical School, Mount Kenya University, 342-01000 Thika, Kenya

*Corresponding Author

Esther Wangui Mandania

\begin{abstract}
The utilization of clinical laboratory test results in the diagnostic decision making process forms an essential aspect of clinical medicine. Inconsistent and unreliable laboratory test results may have severe effects on the health of the patient and society. The aim of this descriptive cross-sectional study was to evaluate the accuracy of most commonly used haemoglobin analysers in determining a standardised reference for low, normal and high haemoglobin values in blood and to compare the manual and automated methods of haemoglobin estimation. A total of 292 laboratories received three samples with low, normal and high haemoglobin concentrations for analysis, after which their results were evaluated for accuracy by comparing with the reference values. Accuracy of the analysers was evaluated using one-way analysis of variance while Coefficient of Variation was calculated as a measure of inter- method variability. Mean deviation from the expected mean of the references reflected the bias of each analyser. Overall $58.4 \%(n=7 / 12), 17.6 \% \quad(n=2 / 12)$ and $25 \%(4 / 12)$ gave accurate, underestimated and overestimated haemoglobin values respectively. Celltac, Humalyzer, Medonic, Mindray, Colourimeter, Hemocontrol and Sysmex produced results that were not significantly different from the reference values $(P>0.05)$. Diaspect and Sahli underestimated while Hemocue, Urit and Mission overestimated Hb values $(P<0.05)$. Automated methods were more precise than the manual methods with Coefficient of Variation for automated, semi-automated and manual methods being $7.08 \%, 7.04 \%$ and $34.26 \%$ respectively. With increasing reliance on the utilization of laboratory test outcomes for clinical decision-making, laboratories mustfrequently participate in External Quality Assessment in order to provide reliable results. Laboratories should embrace automation which gives more accurate and precise results.

Keywords: Haemoglobin, External Quality Assessment, Accuracy, Bias.
\end{abstract}




\section{Introduction}

There are two main elements of a quality assurance programme, namely, IQC and EQA ${ }^{[1]}$. EQA is described by the World Health Organization (WHO), as a method used by an external agency or facility for objectively verifying the performance of a laboratory ${ }^{[2]}$. EQA's main objective is to establish interlaboratories, inter-methods, including interinstrument comparability and agreement with a reference standard as well as detecting systematic errors (bias) ${ }^{[3]}$.

Clinical laboratories of developed countries highly regard quality assurance programmes as one of the key players in quality of laboratory results and thus there is widespread enrolment and participation into these programmes. However, this is not the case in clinical laboratories in resource limited settings. In addition, the general perception that EQA is expensive has hampered extensive laboratory enrolment into these programmes $^{[4]}$.

Medical laboratories have the objective of providing accurate results to facilitate clinical decisions regarding the health of patients. To achieve this objective, laboratories must consider the quality of measurement procedures (MPs) results, engage in routine internal quality control (IQC) assessment, participate in external quality assessment schemes (EQAs) and comparison of their own results with those of other laboratories [5]. Quality assurance in haematology is designed to ensure the reliability of laboratory results.

Laboratory test outcomes may influence $45 \%$ of medical decisions in primary healthcare in East Africa ${ }^{[6]}$. It is therefore important that laboratories generate reliable and accurate results that will guide clinical decision making in order to improve the health of the patient. However, in the resource-limited settings most of the laboratory services provide results of unknown quality ${ }^{[7]}$.

Haemoglobin $(\mathrm{Hb})$ concentration is the most frequently used indicator of anaemia at the individual and population level ${ }^{[8]}$ and is used to screen for anaemia as well as evaluation of responses to intervention programs ${ }^{[9]}$.The $\mathrm{Hb}$ test is precise, easily standardised and may be performed either manually or by using automated hematology analysers ${ }^{[10]}$. The International Committee for Standardisation in Haematology (ICSH) recommends the Drabkin's method as the standard method for determining the $\mathrm{Hb}$ concentration of whole blood ${ }^{[11]}$

In this study, the accuracy and reliability of most commonly used $\mathrm{Hb}$ analysers in Kenyan clinical laboratories was compared to evaluate the results of known samples against reference values. Further the study aimedat comparing the manual and the automated methods of $\mathrm{Hb}$ measurement.

\section{Materials and Methods}

A descriptive cross-sectional study involving a total of 292 laboratories sampled from 21 out of 47 Counties in Kenya and which voluntarily consented to participate in the study was conducted from January to July 2014.Cluster random sampling of the laboratories from the 21 Counties was used so that all categories of laboratories in both public and private hospitals were sampled. The study was based on determination of $\mathrm{Hb}$ concentration in three EQA samples (blood haemolysate) with low (sample A), normal (sample B) and high (sample C) $\mathrm{Hb}$ concentrations, which were prepared in the Central Laboratory at the African Medical and Research Foundation (AMREF), Nairobi, Kenya. $\mathrm{The} \mathrm{Hb}$ concentration (reference values) for the three samples was sample A $(6.2 \mathrm{~g} / \mathrm{dl})$, sample B $(13.6 \mathrm{~g} / \mathrm{dl})$, sample C $(18.1 \mathrm{~g} / \mathrm{dl})$, whose values were assigned using the reference system (Sysmex XS-1000i laboratory)

The samples were placed in leak proof plastic vials which were properly labelled with the unique code numbers. The samples were transported to the laboratories at $4^{0} \mathrm{C}$ in icepacks by carrier. Laboratories were instructed to process the samples within two days after delivery using their current methods of analysis by performing duplicate assays and to analyse the samples in the same manner as routine samples. Alongside the 
study, each laboratory also received a questionnaire so as to collect data on Hb-related analytic information. Laboratories recorded their results in the worksheet provided and the results collected from the laboratories within one week. The results of each laboratory were evaluated for accuracy by comparing with the reference method (Sysmex XS-1000i laboratory).

\section{Study population}

The study covered a total of 292 haematology benches from 292 hospital laboratories sampled from the 21 counties in Kenya. The main study site was the Central Laboratory at African Medical and Research Foundation, Kenya Located in Nairobi, from where the samples were prepared, packaged and distributed to all the participating laboratories.

\section{Inclusion Criteria}

Both public and privately-owned laboratories that perform $\mathrm{Hb}$ measurements and gave free informed consent for participation and are registered by Kenya Medical Laboratory Technicians and Technologists Board (KMLTTB) were included in the study. All methods of $\mathrm{Hb}$ determination being used in the laboratories were applicable.

\section{Exclusion criteria}

Laboratories which are not registered by KMLTTB and which did not give informed consent were excluded from the study.

\section{Ethical Approval}

The study was approved by Kenyatta National Hospital/ University of Nairobi Ethics and Research Committee (Ref: KNH - ERC/A/1).

\section{Data Analysis}

Data were analysed using XLSTAT statistical software (XLSTAT Version 2013.3.03). Using ANOVA, Significant differences $(\mathrm{P}<0.05)$ between means were assessed. Accuracy of analysers was analysed by calculating the difference (denoted as the bias) between the $\mathrm{Hb}$ concentration from the participating laboratory and the reference values. Differences that had pvalues $\leq 0.05$ were considered to be statistically significant.

\section{Results}

A sum of 292 laboratories participated in the study and 27 different analysers were used across all the laboratories (Table 1). Analysers that were used by less than five laboratories were excluded from the analysis, leaving a total of 12 analysers.

Table 1: The Different Analyzers and the Number of Laboratories Using Each Analyser Type

\begin{tabular}{|c|c|c|}
\hline Analyzer & $\begin{array}{l}\text { Number of } \mathrm{Hb} \\
\text { Measurements }\end{array}$ & Number of Labs \\
\hline ABX Micros & 6 & 1 \\
\hline $\begin{array}{l}\text { ACT Diff Beckman } \\
\text { Coulter }\end{array}$ & 18 & 3 \\
\hline BTS 305 & 12 & 2 \\
\hline Celltac & 174 & 29 \\
\hline Cera Check & 18 & 3 \\
\hline Colourimeter & 78 & 13 \\
\hline Coulter Counter & 24 & 4 \\
\hline Diaspect & 306 & 51 \\
\hline Drew & 6 & 1 \\
\hline Easy Mate & 12 & 2 \\
\hline Sahli & 144 & 24 \\
\hline Hb Meter & 12 & 2 \\
\hline Hemocontrol & 264 & 44 \\
\hline Hemocue & 384 & 64 \\
\hline Hichroma & 6 & 1 \\
\hline Humalyzer Junior & 30 & 5 \\
\hline Hybrid & 12 & 2 \\
\hline Kyrot & 6 & 1 \\
\hline Medonic & 30 & 5 \\
\hline Mindray & 60 & 10 \\
\hline Mission & 36 & 6 \\
\hline Pentra ES 60 & 6 & 1 \\
\hline RMS & 6 & 1 \\
\hline Stat & 12 & 2 \\
\hline Sysmex & 48 & 8 \\
\hline UritHb Meter & 30 & 5 \\
\hline Erma & 12 & 2 \\
\hline & 1752 & 292 \\
\hline
\end{tabular}

Applying the $\pm 10 \%$ Allowable Deviation from the Reference Values, most of the analysers gave results that were accurate (comparable with the reference values) for all the three samples A, B and $\mathrm{C}$, with only a few either underestimating or overestimating the $\mathrm{Hb}$ values, as shown in Table 2. 
Table 2: Summary of the Performance of the Analyzers as per the $\pm 10 \%$ Allowable Deviation from the Reference Values

\begin{tabular}{|l|c|c|c|}
\hline Performance Of The Analyser & \multicolumn{3}{|c|}{ No of analyzers And Percentages } \\
\hline & $\begin{array}{c}\text { Sample A } \\
\text { Allowable Error, } \\
\pm 0.62 \mathrm{~g} / \mathrm{dl})\end{array}$ & $\begin{array}{c}\text { Sample B } \\
\text { (Allowable Error, } \\
\pm 1.3 \mathrm{~g} / \mathrm{dl})\end{array}$ & $\begin{array}{c}\text { Sample C } \\
\text { (Allowable Error, } \\
\pm 1.8 \mathrm{~g} / \mathrm{dl})\end{array}$ \\
\hline Underestimated Hb values & $1(8.3 \%)$ & $2(16.7 \%)$ & $2(16.7 \%)$ \\
\hline Comparable with reference values & $8(66.7 \%)$ & $7(58.3 \%)$ & $6(66.6 \%)$ \\
\hline Overestimated Hb values & $3(25 \%)$ & $3(25 \%)$ & $2(16.7 \%)$ \\
\hline TOTAL & $12(100 \%)$ & $12(100 \%)$ & $12(100 \%)$ \\
\hline
\end{tabular}

\section{Accuracy of the various analysers}

The accuracy of $\mathrm{Hb}$ analysers in estimating low, normal and high $\mathrm{Hb}$ concentration is shown in Tables 3, 4 and 5 respectively. The mean $\mathrm{Hb}$ measurements from $22 \%(n=6 / 27)$ of the analysers (Sahli, Mission, Hemocue, Diaspect, Urit and Mission) were significantly different from the mean of the reference values $(\mathrm{F}(27)=17.382$, $\mathrm{P}<0.001$, $) \quad$ while $78 \% \quad(\mathrm{n}=21 / 27) \quad$ (Celltac, Humalyzer Junior, Medonic, Mindray, Colourimeter, Hemocontrol and Sysmex) gave values that were not significantly different from the mean of the reference values $(\mathrm{P}>0.05)$. The most accurate analysers, which gave results within the allowable bias for the three samples A, B and $\mathrm{C}$ were Celltac (bias 0.234, 0.231, 0.600) Colourimeter (bias $0.438,-0.031, \quad-0.708$ ) Hemocontrol (bias 0.048, 0.357, 0.759) Humalyzer Junior ( bias -0.100,-0.600, -0.460) Medonic (bias 0.620, 0.620, 0.440) Mindray ( bias $-0.210,-0.270,0.250$ ) and Sysmex (bias 0.250, 0.175 and 0.150 ) for sample A, Band C respectively as depicted in tables 3, 4 and 5 .

Table 1: The accuracy of the most commonly used analysers in Kenya in estimating low Hb value (6.2 g/dl)

\begin{tabular}{|l|c|c|c|c|}
\hline Analyzer & Value & Standard. error & T statistic & P value \\
\hline Intercept & 6.200 & 0.284 & 21.823 & $<0.001$ \\
\hline Celltac & 0.234 & 0.395 & 0.594 & 0.553 \\
\hline Colourimeter & 0.438 & 0.498 & 0.880 & 0.380 \\
\hline Diaspect & -2.137 & 0.351 & -6.083 & $<0.001$ \\
\hline Hemocontrol & 0.048 & 0.361 & 0.132 & 0.895 \\
\hline Hemocue & 2.167 & 0.339 & 6.397 & $<0.001$ \\
\hline Humalyzer Junior & -0.100 & 0.719 & -0.139 & 0.889 \\
\hline Medonic & 0.620 & 0.719 & 0.890 & 0.374 \\
\hline Mindray & -0.210 & 0.546 & -0.384 & 0.701 \\
\hline Mission & 5.050 & 0.666 & 7.579 & $<0.001$ \\
\hline Sahli & -0.396 & 0.414 & -0.956 & 0.340 \\
\hline Sysmex & 0.025 & 0.594 & 0.042 & 0.966 \\
\hline UritHb Meter & 2.240 & 0.719 & 3.117 & 0.002 \\
\hline
\end{tabular}

Table 2: The accuracy of the most commonly used analysers in Kenya in estimating normal Hb value (13.6 $\mathrm{g} / \mathrm{dl})$

\begin{tabular}{|l|c|c|c|c|}
\hline Analyzer & Value & Standard error & T statistic & P value \\
\hline Intercept & 13.600 & 0.447 & 30.418 & $<0.001$ \\
\hline Celltac & 0.231 & 0.621 & 0.372 & 0.710 \\
\hline Colourimeter & -0.031 & 0.784 & -0.039 & 0.969 \\
\hline Diaspect & -3.988 & 0.553 & -7.213 & $<0.001$ \\
\hline Hemocontrol & 0.357 & 0.568 & 0.628 & 0.530 \\
\hline Hemocue & 2.556 & 0.533 & 4.795 & $<0.001$ \\
\hline Humalyzer Junior & -0.600 & 1.131 & -0.530 & 0.596 \\
\hline Medonic & 0.620 & 1.131 & 0.548 & 0.584 \\
\hline Mindray & -0.270 & 0.860 & -0.314 & 0.754 \\
\hline Mission & 2.917 & 1.049 & 2.782 & 0.006 \\
\hline Sahli & -3.158 & 0.652 & -4.846 & $<0.001$ \\
\hline Sysmex & -0.175 & 0.935 & -0.187 & 0.852 \\
\hline UritHb Meter & 3.760 & 1.131 & 3.324 & 0.001 \\
\hline
\end{tabular}


Table 3: The accuracy of the most commonly used analysers in Kenya in estimating high Hb value (18.1 $\mathrm{g} / \mathrm{dl}$ )

\begin{tabular}{|l|c|c|c|c|}
\hline Analyser & Value & Standard error & T statistic & P value \\
\hline Intercept & 18.100 & 0.540 & 33.498 & $<0.001$ \\
\hline Celltac & 0.600 & 0.751 & 0.799 & 0.425 \\
\hline Colourimeter & -0.708 & 0.948 & -0.747 & 0.456 \\
\hline Diaspect & -8.733 & 0.668 & -13.069 & $<0.001$ \\
\hline Hemocontrol & 0.759 & 0.686 & 1.106 & 0.270 \\
\hline Hemocue & 3.038 & 0.644 & 4.714 & $<0.001$ \\
\hline Humalyzer Junior & -0.460 & 1.367 & -0.337 & 0.737 \\
\hline Medonic & 0.440 & 1.367 & 0.322 & 0.748 \\
\hline Mindray & 0.250 & 1.039 & 0.241 & 0.810 \\
\hline Mission & 1.567 & 1.267 & 1.236 & 0.217 \\
\hline Sahli & -4.900 & 0.788 & -6.221 & $<0.001$ \\
\hline Sysmex & 0.150 & 1.130 & 0.133 & 0.895 \\
\hline UritHb Meter & 4.525 & 1.504 & 3.008 & 0.003 \\
\hline
\end{tabular}

Variation of $\mathbf{H b}$ measurements due to methods A total of $74.32 \%,(n=217)$ of the laboratories used manual methods, $24 \%$ used an automated methods $(\mathrm{n}=70)$ and $1.7 \%,(\mathrm{n}=5)$ used semiautomated methods. ANOVA comparisons revealed that the mean $\mathrm{Hb}$ across the three methods were not significantly different from the mean of the reference values $\left(\mathrm{F}_{(3)}=1.333\right.$, $\mathrm{P}=0.262$, mean of reference value $=12.633$ ). However the CV for the automated and semiautomated methods were similar but was large for the manual method, with a Coefficient of Variation (CV) of $7.08 \%, 7.04 \%$ and $34.26 \%$ respectively (Fig 1).

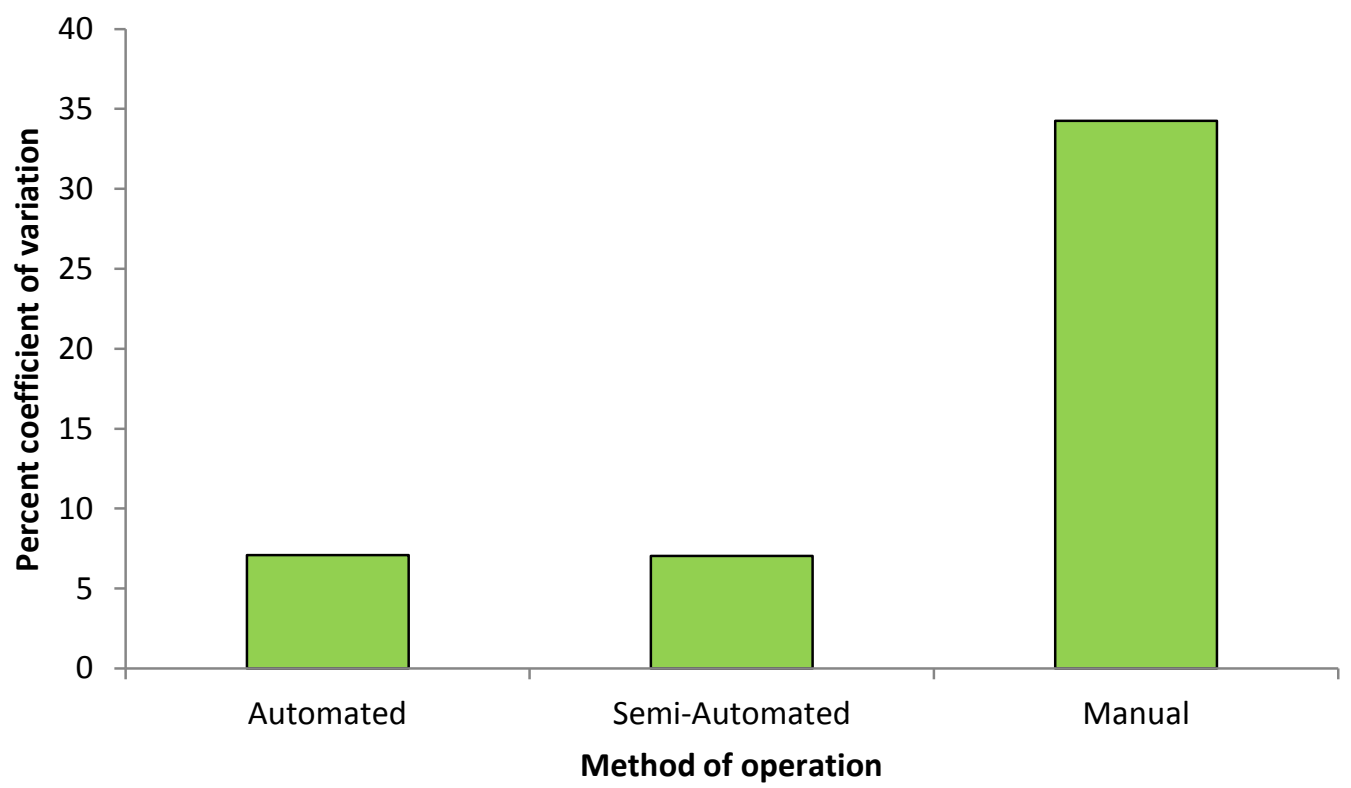

Figure 1: Coefficient of variation around the standard reference as a measure of reliability of the different methods of $\mathrm{Hb}$ analyser operation

\section{Discussion}

Considering that laboratory services play a major role in clinical decision making, then the reliability and quality of laboratory test outcomes is of utmost importance.
Laboratory quality control is intended to identify laboratory errors; and the objective is to guarantee the highest level of accuracy and precision ${ }^{[12,13]}$. Both accuracy and precision of "standard" laboratory measurements are dependent on 
multiple methodologic factors that affect them ${ }^{[14]}$. However, regardless of the method used, it is necessary that the different methods yield values that are as close as possible to the true value. The fact that the analysis of $\mathrm{Hb}$ is dependent on many variables, there is an acceptable range of variation for each of the Hb estimations.

Clinical Laboratories Improvement Amendment (CLIA) has proposed an acceptable range of deviation of $\mathrm{Hb}$ values of $\pm 7 \%$ from the true value and a medically allowed error of $1 \mathrm{~g} / \mathrm{dl}$. The CLIA specification variance is approximately $1.0 \mathrm{~g} / \mathrm{dl}$ for the normal $\mathrm{Hb}$ range of $13-15 \mathrm{~g} / \mathrm{dl}$, while asat the anaemic range of $10 \mathrm{~g} / \mathrm{dL}$ the target variance is $0.7 \mathrm{~g} / \mathrm{dL}^{[15]}$. In other studies a $10 \%$ deviation from the reference method has been used because this variation constitutes only $1 \mathrm{~g} / \mathrm{dL} \mathrm{Hb}$ at the upper range of transfusion consideration $(10 \mathrm{~g} / \mathrm{dL})$ which seems reasonable ${ }^{[16]}$ While all these values may be considered, in this study the $\pm 10 \%$ difference was selected. For every analyser being tested, a difference greater than $\pm 10 \%$ from the reference was considered as probably misleading. In this study, it translates to a deviation of not more than $0.62 \mathrm{~g} / \mathrm{dl}$ for Sample A and not more $1.3 \mathrm{~g} / \mathrm{dl}$ for sample $\mathrm{B}$ which represents the low (critical) range and normal $\mathrm{Hb}$ concentration respectively. Therefore, applying this criterion some analysers surpassed this allowable error gap, with some showing a negative bias while others showed a positive bias. Important to point out is that that these deviations may not be substantial at the normal range of $\mathrm{Hb}$ but very significant at the critical ranges of $6 \mathrm{~g} / \mathrm{dl}$ to $10 \mathrm{~g} / \mathrm{dl}$ where decision is made on whether to or not to transfuse.

Generally, $\mathrm{Hb}$ results acquired from Diaspect and Sahli showed negative bias where both devices underestimated hemoglobin values by more than $2 \mathrm{~g} / \mathrm{dl}$ across the three levels of haemoglobin concentration. The exception was Sahli which at low hemoglobin levels had a tendency to give values comparable to the reference method. The implication of this biases is that underestimation of $\mathrm{Hb}$ concentrations may classify healthy individuals as anaemic and could lead to unnecessary clinical interventions in patients, overtreatment and incurring of additional costs.

The results of this study agree with those of others that have reported underestimation of hemoglobin by Sahli ${ }^{[17,18]}$. The considerable variability in the $\mathrm{Hb}$ values obtained with the Sahli could be due to its inbuilt errors, subjective visual colour comparison, inaccuracy in pipetting of blood, fading of comparator after prolonged use and poor sensitivity and reliability. Contrary to the results presented in this study, Robertson, Lewis, and Osei-Bimpong $^{[19]}$ reported that the $\mathrm{Hb}$ values obtained with Diaspect were comparable with the reference analyser.

On the other hand, this study results show that Hemocue, Urit and Mission consistently overestimated the $\mathrm{Hb}$ values when compared to the reference values. Hemocue overestimated $\mathrm{Hb}$ values by more than $2 \mathrm{~g} / \mathrm{dl}$ and this overestimate appears to increase with increase in the actual $\mathrm{Hb}$ value. This study findings agree with others which have reported overestimation of $\mathrm{Hb}$ values by Hemocue $^{[20,21]}$. However, other studies have reported that Hemocue is accurate and reliabl [22, ${ }^{23]}$. On the contrary, Neufeld et al ${ }^{[24]}$ reported that Hemocue underestimated $\mathrm{Hb}$ values. Due to these contradicting findings on the accuracy and reliability of Hemocue, further studies evaluating the accuracy of Hemocue need to be done. It is important to point out that $\mathrm{Hb}$ values overestimation may result in missing out of the true anemia in a patient where an anemic patient is classified as healthy. This can lead to delays in transfusion, under treatment and consequently increased morbidity.

The findings in this study about Urit agree well with those of Jitthai ${ }^{[25]}$ who reported that Urit gave significantly higher $\mathrm{Hb}$ values than the automated blood analyser. The biggest variation with Urit seen at low and high $\mathrm{Hb}$ concentrations may imply that, laboratories use calibrators for the normal $\mathrm{Hb}$ concentration only without including calibrators for the low and high Hb levels.

This study findings showed there is considerable variation of $\mathrm{Hb}$ values when using manual 
methods in comparison with automated methods and that automated methods have higher precision than the manual methods. This study results agree with those of Fink et $a l^{[26]}$. Inherent errors in the manual methods such as inaccuracies in dilutions, pipetting and improper mixing of samples, and errors caused by the observer would be the causes of this great variation. These apparent differences in $\mathrm{Hb}$ results obtained by different laboratories using different analysers/methods can be addressed by validation of analysers and harmonization of methods for $\mathrm{Hb}$ measurement in order to achieve inter-device and inter-method comparison of $\mathrm{Hb}$ results.

\section{Conclusion}

Celltac, Humalyzer Junior, Medonic, Mindray, Colourimeter, Hemocontrol and Sysmex produced results that are reasonably accurate, however Diaspect and Sahli underestimated $\mathrm{Hb}$ while Hemocue, Urit and Mission overestimated $\mathrm{Hb}$ when compared to the reference values. The manual methods generally showed lower precision when compared to automated methods. There is need for policy guidelines on validation, standardisation and approval of all analysers of $\mathrm{Hb}$ measurement being used in the Kenyan clinical laboratories in order to achieve interlaboratory and inter- methods comparability of results. Laboratories should gradually replace manual methods with automated methods of $\mathrm{Hb}$ measurement which are more accurate and reliable.

Inter-laboratory quality assurance programs should be encouraged by all the relevant bodies and authorities such as KMLTTB and the Ministry of Health. In addition, government at all levels that is national and county governments should be primarily responsible for instituting formal EQAs across the country in order to continually improve laboratory performance and strengthen health care services.

\section{Acknowledgements}

We acknowledge the support received from AMREF Nairobi, Kenya (EAREQAS), in particular Dr. Jane Carter and Mr. Stephen Munene for allowing the preparation of the samples in the Central Laboratory, AMREF, Nairobi. We also express our sincere gratitude to Dr. Patrick Chiyo for performing statiscal analysis. We are also grateful to all the laboratory managers of all the laboratories that consented to participate in this study and the laboratory staff working in these laboratories.

\section{References}

1. Sah, S.P., Raj, G.A. and Prakash, M.B. Quality assurance programme in haematology at a teaching hospital in the eastern region of Nepal. Indian J Pathol Microbiol 1999;42 (2):145-149.

2. World Health Organization. Overview of external quality assessment (EQA): module 10, content sheet 10-1. Geneva, Switzerland: WHO; 2011 Available at: http://www.who.int/ihr/training/laboratory _quality/10_b_eqa_contents. pdf.

3. Cheesbrough M. District Laboratory Practice in Tropical Countries, Part -2 . Low price editions. United Kingdom: Cambridge University Press, 2000: 267270.

4. Peter T, Badrichani A, Wu E, Freeman R, Ncube B, Ariki F, et al. Challenges in implementing CD4 testing in resourcelimited settings. Cytometry B Clin Cytom 2008;74B:S123-S130.

https://doi.org/10.1002/cyto.b.20416.

5. James D, Ames D, Lopez B, Still R, Simpson W, Twomey P. External quality assessment: best practice. J Clin Pathol 2014; 67:651-655.

6. Carter JY, Lema OE, Wangai MW, Munafu CG, Rees PH, Nyamongo JA. Laboratory testing improves diagnosis and treatment outcomes in primary health care facilities. Afr J Lab Med 2012;1(1):8 
7. Petti CA, Polage CR, Quinn TC, Ronald AR, Sande MA. Laboratory medicine in Africa: a barrier to effective health care. Clin Infect Dis 2006;42:377-82. https://doi. org/10.1086/499363.

8. WHO. Haemoglobin concentrations for the diagnosis of anaemia and assessment of severity. vitamin and mineral nutrition information system. Geneva, 2011.

9. Morris SS, Ruel MT, Cohen RJ, Dewey KG, de la Brière B, Hassan MN. Precision, accuracy, and reliability of hemoglobin assessment with use of capillary blood. Am J ClinNutr 1999;69(6):1243-8.

10. Barbara H, Anna P, Norma J.Basic Medical Laboratory Techniques. 4th Ed. Albany, NY; Delmar Publlishers, 2000:

11. World Health Organization.Worldwide prevalence of anaemia 1993-2005:WHO Global Database on Anaemia. Geneva: World Health Organization; 2008. Available from: [http://whqlibdoc.who.int/publications/200 8/9789241596657_eng.pdf]

12. Rauf M, Hanan A. Quality assurance considerations in chemical analysis. Qual Ass J 2009;12: 16-21.

13. Agarwal R. Quality-Improvement Measures as Effective Ways of Preventing Laboratory Errors. Lab Medi2014;45: 8088.

14. Berkow L. Factors affecting hemoglobin measurement. J Clin Monit Comput 2013; 27(5):499-508. doi: 10.1007/s10877-0139456-3.

15. Medicare, Medicaid and CLIA programmes; regulations implementing the clinical laboratories improvement amendments of 1988 (CLIA) -FFCA. Final rule with comment period. Fed Regist 1992; 57(40):7002 - 186.

16. American Society of Anesthesiologists Task Force on Perioperative Blood Transfusion and Adjuvant Therapies. Practice guidelines for perioperative blood transfusion and adjuvant therapies: an updated report by the American Society of Anesthesiologists Task Force on Perioperative Blood Transfusion and Adjuvant Therapies. Anesthesiology 2006;105:198-208

17. Patil P, Thakare G,Patil, S. Variability and Accuracy of Sahli's Method in Estimation of Haemoglobin Concentration. Natl J Integr Res Med 2013;4 (1):38-44.

18. Kapil U, Tandon M, Pathak P, Dwivedi SN.Comparison of haemoglobin values obtained by Haemocue and Sahli's methods. Ind J Public Health2002; 46 (1):28 -30.

19. Robertson L, LewisD, Osei-Bimpong A. Utility of the Diaspect Haemoglobinometry System as Point- OfCare Analyzer. J Near Patient Test Technol 2011; 10 (1):17-21.

20. Mohanram M, Ramana-Rao GV, Sastry JG. A comparative study on prevalence of anaemia in women by cyanmethemoglobin and hemocue methods.Indian $\mathbf{J}$ Community Med 2002;27 (2):58-61.

21. Bhaskaram P, Balakrishna N, Radhakrishna KV, Krishnaswamy K. Validation of haemoglobin estimation using hemocue. Indian J Pediat2003; 70:25-28.

22. Sari M, De Pee S, Martini E, Herman S, Sugiatmi,BloemMW, Yip R. Estimating the prevalence of anaemia: a comparison of three methods. Bull World Health Organ 2001; 79(6):506 - 511.

23. Rechner IJ, Twigg A, Davies AF,Imong S.Evaluation of the hemocue compared with the coulter STKS for measurement of neonatal haemoglobin. Arch Dis Child Fetal Neonatal Ed 2002; 86(3): F188 189.

24. Neufeld L, Garcia-Guerra A, SanchezFrancia D, Newton-Sanchez O, RamirezVillalobos MD,Rivera-Dommarco J. Haemoglobin measured by hemocue and a 
reference method in venous and capillary blood: a validation study. Salud Publica Mex 2002; 44:219 - 227.

25. Jitthai S.A comparison of haemoglobin measurement between haemoglobinometer (POCT) and automated blood cell analyzer in hemodialysis patient. J Med Technol Assoc of Thai 2012; 40 (3):4332 - 4337.

26. Fink NE, Fernandez Alberti A,Mazziota D.External assessment of analytic quality in haematology: a necessity in Latin America. Rev Panam Salud Publica 1997; 2(3):181-188. 